\title{
Exploring Information-Triage: Speculative Interface Tools to Help College Students Conduct Online Research
}

\author{
Liese Zahabi \\ Weber State University \\ Ogden, UT, USA \\ liesezahabi@weber.edu
}

\begin{abstract}
In many ways, the promise of the Internet has been overshadowed by a sense of overload and anxiety for many users. The concept of informationtriage may help mitigate this issue. Information-triage is the process of sorting, grouping, categorizing, prioritizing, storing and retrieving information in order to make sense and use of it. This study examines the role of design in the online search process, connects it to the nature of human attention and the limitations of working memory, and suggests ways to support users with an information triage system. The study centers on a set of three speculative online search interfaces and user-testing sessions conducted with college students to explore the possibilities for information-triage.
\end{abstract}

Keywords: Information-triage, interface, interaction, search, user-testing, visualization, metaphor.

\section{Introduction}

Online search has become a part of everyday life. College students in particular conduct scores of searches, in their studies and outside of school. Search engines like Google give users access to previously unimaginable amounts and types of information, but the ways in which search results are organized and presented can often obfuscate as much as inform. The production and publication of online material has become increasingly accessible and affordable, creating a confusing glut of information often leading to a sense of overload and anxiety [13].

Information overload is not a new problem [12]. What has changed in the last few decades is the ease of access to an exponential barrage of information. According to Shirky [9] what we are experiencing today is actually filter failure. Filters that developed over the last few hundred years to deal with large amounts of information have started to break down as the Internet has moved our information culture from a process of top-down edited publication to one of bottom-up open-source dissemination. Design can help by developing better tools and systems to help users understand and filter the information they encounter online. One method is information-triage.

The concept of information-triage is derived from the medical term, and migrated to the computing and business world as tasks and jobs became increasingly complex 
and data-driven. Information-triage is the process of sorting, grouping, categorizing, prioritizing, storing and retrieving information in order to make sense and use of it. Lunenfeld [6] describes the notion: "Info-triage is more art than science, a practice that involves the weighing of options and the measuring of time. We tend to think of time in relation to efficiency, but info-triage is about more than job performance, it is a practice devoted to mindfulness... [it] is not so much about efficiency as the culling of the distraction in the search for meaning." In this context, information-triage is not merely a sorting technique, but also a type of curation - a method in which users can control the information they encounter in whatever way is most meaningful for the task at hand.

Current popular search engines, especially Google, often excel at cataloging the web and returning increasingly accurate and relevant results. But the functionality and visualization of both the interface and results is inadequately developed, and in many cases, can be a hindrance to a user's cognitive abilities and needs. When faced with a homogeneous screen of text, most users have trouble discerning among the returned results, because visual, spatial, cognitive and sensemaking cues are not utilized in a meaningful way.

\section{Related Work}

\section{Working Memory}

Our ability to process complex information is related to how well we can focus our attention. This focus is directly linked to the capacity and limitations of our working memory [1], which is "a limited capacity temporary storage system that underpins complex human thought". Working memory allows the brain to actively hold and temporarily capture information. It is part of what makes attentional control, focusing on one object or concept while ignoring others, possible. Working memory has a limited capacity. At some point, it becomes full. New pieces of information can be taken in but only through the loss of something else.

The limitations of working memory have many implications for the task of searching online. Attempting to make sense of multiple search results at once can fill the capacity of working memory, creating a sense of unease or confusion for users. This is specifically difficult because of the way most search engine interfaces are designed. The structure of the pages, the use of minimally styled lists of text and the repetitive similarity of the pages do not allow users to effectively discriminate relevant results. Most search interfaces found online fail to address this cognitive limitation.

\section{Sensemaking}

Sensemaking is a cognitive process that has been studied in several different knowledge domains including medicine, policy-making and the intelligence community. Sensemaking allows humans to make sense of complex information and data encountered in the world around them. [8]

Several examples of interface prototypes that incorporate sensemaking research have been designed. Examples include interfaces: to help military experts make 
decisions [7]; to support geographic analysts [11], and to engage self-directed student learners in the process of completing tasks related to education [3]. These interfaces utilize spatial cues, cordoning sections off into different boxes for different task purposes. The designs also integrate drag-and-drop techniques to help users intuitively understand the movement and sorting of information. Two of the interfaces integrate maps and charts, enabling users to plot information points using these schematics.

Overall, these examples do not address the need for triage to occur at the site of the search engine itself, where raw data is being initially chosen and collected. While the systems do acknowledge the creation of spatial understandings of visual data, the designs do not go much further beyond organizing the space into multiple divisions and boxes. Other types of visualization and organization could be employed to further address the cognitive needs of users.

\section{Other Interfaces and Studies}

Interfaces have been developed and tested to focus on the effects clustering of web pages on mobile devices [5], and navigation via index pages and guided tours [14]. User-testing has also been conducted to explore students' search performance related to implicit and explicit search strategies [10]. While these interfaces and studies are looking at specific audiences and their online habits, they do not address the visualization or organization of search results in any meaningful way.

\section{Study Methodology}

A series of three speculative interface prototypes were designed and developed for this study, (shown in Figure 1). These interfaces explore a range of system- and usercontrol, utilizing various methods of information-triage to provide avenues for visual organization and categorization. The designs are intended to exist within an Internet browser, and would incorporate results generated by Google. The three interfaces are: the Narrative Guide, Mise en Place, and the Intelligent Path. (Animations demonstrating the interfaces and additional information can be found here: http: / / www. zahabidesign. com, under Information-Triage Research).

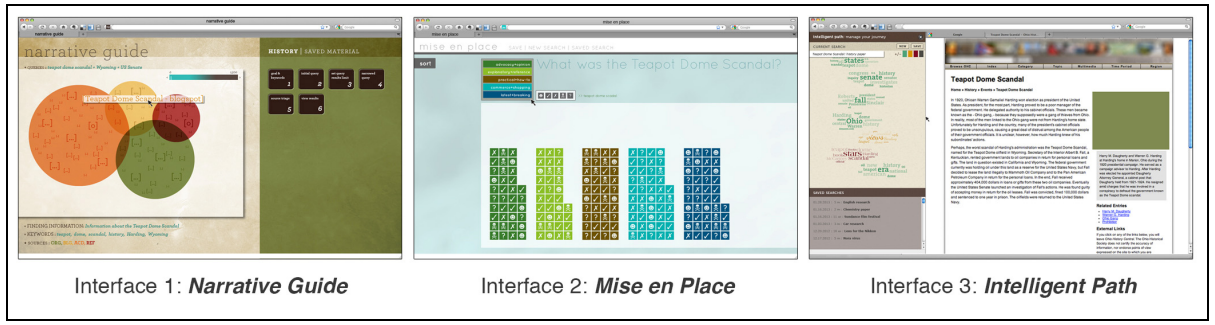

Fig. 1. The three interface designs 


\subsection{The Narrative Guide}

This interface is designed to take the user step-by-step through the search journey. It is partly based on the idea of Choose Your Own Adventure books, in which the user is heavily guided through a process, prompted with structured choices constantly and consistently. The system keeps track of the user's search and suggests options and alternatives as a collaborative coach [2]. The Narrative Guide prompts the user, helping her to develop a sound search strategy based on her stated goals and motivations for the search.

The look, feel, and function of the system are all meant to inspire comfort and trust. Even the language of the system has been crafted to offer reassurance and promote focus. Users of the Internet have become accustomed to fast-acting and highly responsive websites and interfaces. The Narrative Guide asks our user to slow down, to be thoughtful about her query choices, and to be methodical when viewing results. It also displays those results in a visual and information-rich way, which can create affordances for different kinds of understanding and connection making.

\subsection{Mise en Place}

This interface is based on the idea of mise en place (French for "everything in its place") that chefs and cooks utilize while cooking. Essentially, mise en place is a methodical way to organize all elements and implements before the act of cooking begins, enhancing competency and providing efficiency through established expectations. The Mise en Place system allows the user to organize and state the purpose of her search, and then displays coded results. These results are labeled according to large categories generated using keywords and source types, and a gradient of credibility generated by other users of the system. The interface is meant to create a friendly and comforting environment that will allow users to make sense of search results and find focus.

The Mise en Place interface encourages the user to sort through her search results. She can easily conduct mass sorts using the basic category and credibility menus, and she can discard types of results that are note relevant to her search. The user can also utilize a personal Sort space, creating categories and affinities that fit individual criteria. By providing a simple set of tools and functions, the system allows for a wide range of interactions and experiences.

\subsection{The Intelligent Path}

Both Bing and Google offer search history functions, with features that include filtering and chronological searching. This system builds on this idea, creating an Intelligent Path to help a user keep track of a search. The interface functions as a plug-in to a typical Internet browser and operates as a collapsible drawer that houses the user's material. The top portion contains the user's Intelligent Path, along with functions for saving and creating a new search. The bottom portion contains a list of saved searches. 
The interface is meant to be unobtrusive and ambient as the user conducts a routine search, keeping track of visited sites and creating a chronological path to re-visit when desired. The user can utilize the interface if and when she needs to, turning it on and allowing it to collect her movements, and then visiting the data at a later time.

The system visualizes each website as a typographical tag-cloud, triaging the content into a set of the ten most-used words. These key words allow a user to quickly ascertain whether the site content is relevant to her search and provides serendipitous juxtapositions and meaningful connections among words she had not initially thought to search for. Each tag-cloud is color and typeface coded to indicate type of source.

\subsection{Paper Prototypes}

The three interfaces described above were converted into paper prototypes for use during the second phase of user-testing. Participants were asked to simulate a typical search task while they interacted with the prototypes. Some elements of the interface were preemptively chosen and printed as part of the paper prototype and some elements were left open for interpretation.

\subsection{Phase One: Baseline User-Testing}

A series of ten baseline user-testing studies were conducted. College students were chosen based on a campus-wide call for participation. Both female and male participants were chosen. Participants were asked to fill out a short survey to establish familiarity and comfort level with the technology used in the study and with searching online in general. Participants completed a form asking them to locate facts and information about a specified research topic. The form also asked participants to compare and contrast aspects of the topic in order to make decisions.

All participants conducted online search on the same iMac and iPad, and all sessions were recorded using video cameras, screen recording, and mouse tracking software. Obtrusive and unobtrusive observation was conducted; the researcher observed the participant's behavior and asked questions to encourage the participant to describe search methods, techniques, behaviors and experience.

\subsection{Phase Two: Interface User-Testing with Paper Prototypes}

A second series of eight user-testing studies were conducted, asking participants to engage with paper prototypes of the three information-triage interfaces described above. College students were chosen based on a campus-wide call for participation. Both female and male participants were chosen. Participants were asked to fill out a short survey to establish familiarity and comfort level with computer technology, and with searching online in general.

As before, the tests were conducted using obtrusive and unobtrusive observation, and the sessions were recorded and analyzed. The researcher gave the participants a typical and targeted search task: pretend you are taking an American History class and your professor has asked you to write a five-page research paper about the Teapot 
Dome Scandal, you will pretend to use these interfaces to conduct your online research. None of the students had any familiarity with the chosen research topic. Participants were encouraged to indicate frustration and interest, and to give feedback/make comments freely throughout the study.

\section{$4 \quad$ Results}

\subsection{Surveys}

Eighteen students participated in the study. The age of the participants ranged from 18 to 40 , and the average age was 27 . Participants claimed to have been using computers for an average of fifteen years, and stated that they were "very comfortable" using them.

Most participants indicated that online search engines are important to them, with 61 percent responding "very important" and 28 percent responding "somewhat important". When asked about the kinds of tasks participants use computers to execute in a typical day, 100 percent responded "searching/researching using search engines." The other responses are listed in Table 1.

Table 1. Tasks participants stated they use computers to execute in a typical day

\begin{tabular}{|l|l|}
\hline Response & Tasks (users were allowed to choose multiple tasks) \\
\hline $100 \%$ & Searching/researching using search engines \\
\hline $94 \%$ & Socializing with friends and other networks \\
\hline $89 \%$ & Communication: IMing, chatting, e-mail, Skype, etc. \\
\hline $83 \%$ & Entertainment \\
\hline $78 \%$ & Work or Productivity \\
\hline
\end{tabular}

78 percent of participants indicated they felt "very comfortable" using online search engines, three indicated they felt "somewhat comfortable," and one participant indicated they felt "neutral." When asked which search engine they prefer to use, all but one of the participants indicated Google. Participants were also asked what they typically use a search engine to do and how they decide what to type into the search box when they use online search engines. The responses to these questions are found in Table 2 and Table 3.

Table 2. Responses to what participants typically use a search engine to do

\begin{tabular}{|l|l|}
\hline Response & What do you typically use a search engine to do? \\
\hline $89 \%$ & Find quick answers to simple questions \\
\hline $83 \%$ & Academic research for school \\
\hline $72 \%$ & To quickly get to a website (instead of typing address into the URL bar) \\
\hline $67 \%$ & Do research about products or items to purchase \\
\hline $33 \%$ & Research related to work/job \\
\hline
\end{tabular}


Table 3. Responses regarding how participants decide what to type into a search box

\begin{tabular}{|l|l|}
\hline Response & How do you decide what to type into the search box? \\
\hline $72 \%$ & Just start typing \\
\hline $39 \%$ & Type in one thing and then use the results to help type in something else \\
\hline $22 \%$ & Think about what to type for a few minutes first \\
\hline $.06 \%$ & Write down a few things and then start typing \\
\hline
\end{tabular}

\subsection{Phase One: Baseline User-Testing}

Based on patterns revealed in the phase one study, students are very concerned with issues of relevance and trustworthiness when it comes to searching for source material online. Most participants were quickly able to locate basic facts and information during the sessions; more complex searches took longer and many students expressed frustration when asked to make complicated or ambiguous comparisons. Many participants indicated suspicion about unreliable sources, and indicated concern about locating sources that were "correct," "reliable," or "believable." When asked how participants know which sources are reliable, most mentioned type of web address (.org or .gov being most acceptable) and whether the website "looks professional." When pressed regarding the visualization of a website, participants were unable to tangibly describe why one website looks more "professional" than another. This led to many discussions around the idea "I know it when I see it."

When asked, "How would you make online search better?" many students answered with variations of, "If the computer could just read my mind." Often these discussions were actually revolving around ideas of relevancy and trustworthiness, as students struggled with sifting through the large number of results returned during a typical online search. Most participants indicated frustration and impatience when the answer to a question or query wasn't immediately apparent or when they were confronted with conflicting results.

\subsection{Phase Two: Interface User-Testing with Paper Prototypes}

Overall, students responded positively to the main concepts and features in the three interface designs. Many fine points of the user interface (UI) design itself were confusing or uncomfortable and participants made several excellent suggestions for improvement. The researcher focused on the actual behavior of the participants along with the verbal feedback and comments.

\section{The Narrative Guide}

Most of the participants stated that this interface was easy to use and felt "friendly" and "comfortable." Most liked the overall design and layout, stating that it was "colorful," "intuitive," and "clean/uncluttered." All the participants responded positively to the fact that the system kept track of the search process for them, and that it would allow for saved searches. Participants also liked that the system would allow them to search for specific categories and types of material. 
Many participants responded positively to the "results narrowing" step of the process, in which the interface prompts the user to choose a number of results, and provides a small chart visualizing how each search compares to the previous, and to the chosen target. "I like [setting] the goal of how many results you want...it gives you a better perspective of how much stuff is out there (S04)".

Some participants liked the considerable amount of prompting by the system, "I really like that it just walks you through everything, and...it lists everything that you've already done (S02)", "I liked how it would prompt you...if you wanted to just look at the broad picture, or if you wanted to narrow it down (S08)". Some participants thought there should be a way to bypass some of the prompting, "It did kind of seem like there was a lot of steps maybe that we could bypass (S08)", "I liked and didn't like that there was so many options and steps (S09)". Some students thought there were too many prompts and steps in the initial part of the interface, and many stated that the system should allow an advanced user to bypass the beginning steps of query making.

\section{Mise en Place}

Participants generally identified whether they were a "visual or verbal person" as they gave feedback regarding this interface. Some participants enjoyed the color and symbol coded results, and some wished the results were text based "because it feels more familiar." Most participants responded positively to the rating system itself, "Because then you know based on what other people thought and found useful, and sort that into categories and not have to go through junk to get what you need...that's faster (S06)".

However, the visualization of the rating system was confusing to many of the participants, "I like to see...words, more than just the symbols. For some people, I think the symbols probably would work (S08)", "You kind of lost me at the symbols rather than the actual content...I guess the different symbols were confusing to me (S10)".

Most participants felt the sorting functions were useful, but thought the UI controls were awkward and not intuitive. Some participants liked the personal sort space, describing it as "... way better than just having a place where you just have it written down (S02).” Many found the concept useful, but the visual execution felt confusing.

\section{The Intelligent Path}

Most of the participants liked that this interface operates alongside a normal search engine, "I really like how it's next to the actual website because it makes looking at the website a lot easier so you don't have to have a whole bunch of tabs open (S02)", "I liked this...instead of having to have a paper to put your research on, it saved it here, so that worked (S05)". Participants liked that the system automatically kept track of search history, but most felt that the visualization was a bit clunky and the interaction was not very intuitive. Some found this interface far less useful than the other two, "To be honest, it seems kind of unnecessary (S09)".

Participants were split between those who responded positively to the use of verbal language, and those who wanted more iconic or visual elements, "I liked being able to see the words part. And that from each article it...took...keywords (S08)", "I think 
I'm more visual, because when I see words...it's more confusing to me because I have to read it...the icons are probably easier for me (S06)". In general, students had fewer comments regarding this interface, but many thought ideas from the other two interfaces could be integrated into a collapsible drawer.

\section{Discussion}

Participant preferences were split between the Narrative Guide and Mise en Place interfaces. Some preferred Mise en Place because it was quicker and used icons, and included a user-generated rating system. Several participants stated that they preferred the Narrative Guide to Mise en Place, because it allowed them to develop a search strategy, provided prompts, and kept track of the steps in the search. However, even the participants who preferred the Mise en Place interface thought that it felt unfamiliar and confusing at first, "I didn't really know what I was looking at at the beginning...I didn't really know how to interact with it. Once I figured it out it was awesome (S02)".

Many of the participants categorized themselves as either "visual" or "verbal," and often either "analytical" or "artistic," and used those terms to explain their responses to the interfaces. Research regarding how these designations affect the perception of interface design and online search results will be integrated into subsequent user-testing studies.

Typically, search engines return results that look identical, purely language-based and neutral. The interfaces in this study attempt to color these same search results using sets of meaningful criteria, allowing a user to understand specific aspects of those results before choosing to examine them in detail. The interfaces allow users to see and understand more results at once, encouraging users to compare and contrast and make connections among results across broad categories and source types.

The study demonstrates that some students struggle with current search engines and academic research tools, and can be frustrated and confused when executing online search tasks. The preliminary user-testing shows that metaphoric interfaces utilizing the concept of information-triage can engage college students to help support their online research efforts.

\section{Conclusions and Future Work}

Developing systems that incorporate information-triage can help to engage users in meaningful ways. The creation of interfaces that incorporate information-triage, that feel intuitive, and take into account issues of relevance and trustworthiness in a less ambiguous way, and that allow for easier comparisons and connections among search results can all help to meet users where they are cognitively and culturally.

The three interfaces in this study begin to address these concerns and elements of each design were successful. However, the unfamiliar nature of the metaphoric structures proved confusing in several places, and participants were split between wanting visual/icon based elements and verbal/text based elements.

A more successful design will do the following: 
- Combine useful elements from all three designs

- Combine visual and verbal information so that both "types" of students are served and engaged by the UI

- Better accommodate advanced users, providing advanced search options and ways to skip steps when desired

- Include functionality for academic research tasks like citing and tracking sources

- Allow flexibility so users can control the level of prompting and pace of the search

- Find different ways to visualize the spatial and organizational metaphors being used, creating a more intuitive and understandable system

Future work will include the design of a new interface implementing the ideas listed above and another phase of user-testing. A larger sample size will be used for this next phase of testing, and both paper prototypes and animated digital prototypes will be utilized. This phase will focus on how users intuitively sort and categorize content, and ways the interface designs can help support those kinds of tasks.

\section{References}

1. Baddeley, A.: Working Memory, Thought, and Action. Oxford University Press, New York (2007)

2. Brajnik, G., Mizzaro, S., Tasso, C., Venuti, F.: Strategic help in user interfaces for information retrieval. Journal of the American Society for Information Science and Technology 53(5), 343-358 (2002)

3. Butcher, K.R., Sumner, T.: Self-Directed Learning and the Sensemaking Paradox. In: HCI, vol. 26, pp. 123-159 (2011)

4. Klingberg, T.: Overflowing Brain: Information Overload and the Limits of Working Memory. Oxford University Press, New York (2009)

5. Liu, J., Yu, C., Xu, W., Shi, Y.: Clustering Web Pages to Facilitate Revistation on Mobile Devices. In: Proc. IUI 2012, pp. 249-252 (2012)

6. Lunenfeld, P.: The secret war between downloading and uploading: tales of the computer as culture machine. MIT Press, Cambridge (2011)

7. Ntuen, C.A., Park, E.H., Gwang-Myung, K.: Designing an Information Visualization Tool for Sensemaking. IJHCI 26(2-3), 189-205 (2010)

8. Pirolli, P., Russell, D.M.: Introduction to this Special Issue on Sensemaking. In: HumanComputer Interaction, vol. 26, pp. 1-8 (2011)

9. Shirky, C.: It's Not Information Overload, http: / /web2expo.blip.tv/file/1277460

10. Tsai, M., Hsu, C., Tsai, C.: Investigation of High School Students' Online Science Information Searching Performance: The Role of Implicit and Explicit Strategies. J. Sci. Educ. Technol. 21, 246-254 (2012)

11. Tomaszewski, B., Blandford, J., Ross, K., Pezanowski, S., MacEachren, A.M.: Supporting Geographically-Aware Web Document Foraging and Sensemaking. In: Computers, Environment and Urban Systems, vol. 35, pp. 192-207 (2011)

12. Wright, A.: Glut: Mastering Information Through the Ages. Joseph Henry P, Washington DC (2007)

13. Wurman, R.S.: Information Anxiety. Doubleday, New York (1989)

14. Yang, T., Ferati, M., Bolchini, D.: Navigating by Index and Guided Tour for Fact Finding. In: Proc. SIGDOC 2012, pp. 181-190 (2012) 